\title{
Mortalidade materna: 75 anos de observações em uma Maternidade Escola
}

\author{
Maternal mortality: 75 years of observations in a teaching maternity hospital \\ Amaury Teixeira Leite Andrade ${ }^{1}$, Martha de Oliveira Guerra ${ }^{2}$, Gilberto Nogueira de Andrade ${ }^{3}$, \\ Dimas Augusto de Carvalho Araujo ${ }^{4}$, José Paixão de Souza ${ }^{5}$
}

\section{RESUMO}

Objetivo: avaliar as causas de todas as mortes maternas ocorridas no período de 1927 a 2001 entre 164.161 pacientes, internadas no Serviço de Obstetrícia da Universidade Federal de Juiz de Fora. MG. Métodos: estudo retrospectivo das 144 mortes maternas que ocorreram na maternidade em 75 anos, com um total de 131.048 nascidos vivos, utilizando todos os prontuários de pacientes, avaliados pela história clínica e dados da certidão de óbito (não foram realizadas necropsias). Foram registrados a idade, paridade, tempo de gestação, complicações, momento e causas de morte, estabelecendo-se o índice de mortalidade materna (IMM) hospitalar por cem mil nascidos vivos. Análise estatística pelo teste do $\chi^{2}$ e pela técnica de amortecimento exponencial $(\alpha=0,05)$. Resultados: de 1927 a 1941 o IMM foi de 1544, entre 1942 e 1956 houve redução para 314 ( $p<0,001)$ e de 1957 a 1971 decresceu para 76,4 por cem mil nascidos vivos ( $\mathrm{p}<0,001)$. No entanto, desde 1972 tem se mantido estável (IMM=46 nos últimos 15 anos, p=0,139). As mortes maternas mais freqüentes ocorreram entre 15 e 39 anos, em nulíparas com gestação a termo, e no puerpério imediato (53\%). Causas obstétricas diretas foram responsáveis por 79,3\% dos casos e indiretas em 20,7\%. Analisando as causas de mortes, verificou-se que no primeiro período as causas obstétricas diretas mais freqüentes em ordem decrescente, foram a infecção puerperal, eclampsia e ruptura uterina intraparto; no segundo período, foram a hemorragia pré-parto e eclampsia, e entre 1977 e 2001, as hemorragias, abortos e pré-eclampsia. A análise dos últimos 15 anos mostrou que não houve morte por pré-eclampsia/eclampsia nem infecção puerperal e as principais causas foram hemorragia periparto, aborto e obstétricas indiretas. Relacionando a mortalidade materna por tipo de parto pelo risco relativo associado à cesárea e/ou parto vaginal, verificou-se que, quando a cesárea é indicação inevitável, o risco a ela associado é menor (risco relativo $=0,6$ ) que o de parto por via vaginal. Conclusões: apesar da redução ao longo dos 75 anos, a mortalidade materna, de 46 por 100 mil nascidos vivos, ainda é muito elevada, não havendo decréscimo significativo desde 1972, e muitas mortes são evitáveis. Hemorragias são atualmente as causas mais freqüentes de morte materna. A mortalidade materna por aborto tem aumentado de maneira alarmante e o planejamento familiar efetivo é indispensável.

PALAVRAS-CHAVE: Mortalidade materna; Aborto; Hemorragia; Pré-eclâmpsia; Eclampsia; Infecção puerperal; Estudos retrospectivos

\section{ABSTRACT}

Purpose: to evaluate all maternal deaths that occurred between 1927 and 2001, among 164,161 patients admitted to the Maternidade Therezinha de Jesus, the obstetrical service of the "Universidade Federal de Juiz de Fora", Brazil. Methods: a retrospective study of 144 maternal deaths that occurred in the maternity hospital in 75 years, with 131,048 live births in the same period of time, analyzing all patients's records regarding their clinical history and data from death certificates. Autopsies were not performed. Data obtained were age, parity, gestation length, complications, moment, and causes of death. The index of maternal mortality (IMM) period 100 thousand live births was utilized. For statistical analysis the $\chi^{2}$ test and the exponential smoothing technique were used $(\alpha=0.05$ ). Results: IMM decreased from 1544 in the period 1927-1941 to 314 ( $p<0.001)$ between 1942 and 1956 and from 1957 to 1971 it was reduced to 76.4 per 100 thousand live births $(p<0.001)$. Nevertheless, since 1972 there was no further significant improvement (IMM=46 in the last 15 years, $p=0.139$ ). Maternal mortality was more frequent in the

Maternidade Therezinha de Jesus, Centro de Biologia da Reprodução, Universidade Federal de Juiz de Fora - UFJF - Juiz de Fora (MG), Brasil.

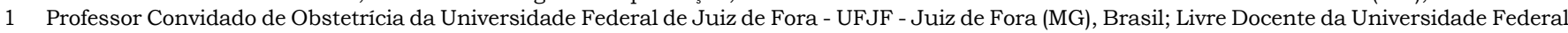
do Rio de Janeiro - UFRJ - Rio de Janeiro (RJ), Brasil; Diretor Clínico da Maternidade Therezinha de Jesus - MTJ - Juiz de Fora (MG) - Brasil.

2 Professora, Pesquisadora do Centro de Biologia da Reprodução da Universidade Federal de Juiz de Fora - UFJF - Juiz de Fora (MG), Brasil.

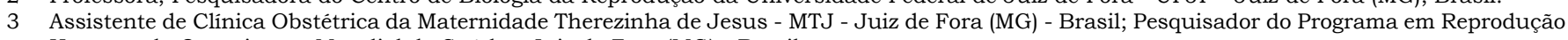
Humana da Organização Mundial da Saúde - Juiz de Fora (MG) - Brasil.

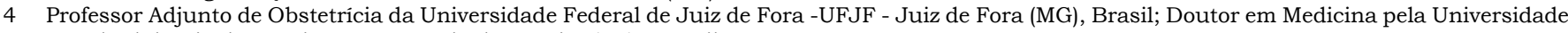
Estadual do Rio de Janeiro - UERJ - Rio de Janeiro (RJ) - Brasil.

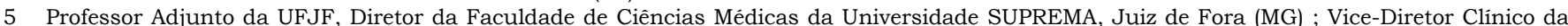
Maternidade Therezinha de Jesus - MTJ - Juiz de Fora (MG) - Brasil.

Correspondência: Amaury T.L. Andrade

Rua Dr. Dirceu de Andrade, 33 - CEP: 36025-330 Juiz de Fora - MG - fax: (32) 3232-4128, e-mail: amandrad@terra.com.br 
15 to 39 years age group, in nulliparous patients with term pregnancies and mostly in the immediate postpartum period (53\%). Direct obstetric causes occurred in $79.3 \%$ and indirect causes in $20.7 \%$ of the cases. Analyzing the evolution of the causes of death, it was found that in the first period of time the most frequent direct obstetric causes in descending order were puerperal infection, eclampsia and uterine rupture, while in the second period they were prepartum hemorrhage and eclampsia, and from 1977 to 2001 hemorrhage, abortion and preeclampsia. Analysis of the past 15 years showed the absence of maternal deaths by either preeclampsia or puerperal infection and the main causes were peripartum hemorrhage, abortion and indirect obstetrical causes. Relating maternal mortality to the type of delivery by the relative risk between cesarean section and vaginal delivery, it was found that when the indication of cesarean section is inevitable its risk is lower (relative risk $=0.6$ ) than through vaginal delivery. Conclusions: despite the reduction along the 75 years of study, maternal mortality of 46 per 100,000 live births is still very high, and there was no significant decrease since 1972. Many deaths are avoidable. Hemorrhage is at present the most frequent cause of maternal death, the decision to intervene should be fast, and a proper indication for a cesarean section is a safe option. Maternal mortality caused by abortion is increasing alarmingly and family planning is essential.

KEYWORDS: Maternal mortality; Abortion; Hemorrhage; Preeclampsia; Eclampsia; Puerperal infection; Retrospective studies

\section{Introdução}

A morte de uma jovem mulher relacionada à sua gravidez é tragédia para sua família e é vergonha para o estado da saúde de um país. Publicação recente da Organização Mundial da Saúde (OMS) informa que a mortalidade materna correlaciona negativamente com o funcionamento do sistema de saúde: quando ela é alta, deve-se concluir que o sistema de saúde não funciona bem ${ }^{1}$. A despeito do reconhecimento mundial de que a morte de mais de 500.000 parturientes a cada ano é inaceitável e que possivelmente poderia ser evitada, mulheres dos países em desenvolvimento continuam a morrer em ritmo similar ao de países mais ricos de décadas atrás. Embora as proporções variem de país para país, geralmente as mulheres morrem de cinco causas maiores que são, em freqüência decrescente: hemorragia, sepse, aborto, eclampsia e parto obstruído $^{2}$. Para a OMS, um dos grandes problemas relacionado à mortalidade materna é a falta de dados estatísticos confiáveis, e, particularmente nos países onde a mortalidade materna é mais elevada, os problemas de sub-registro e de classificação errônea são endêmicos. A OMS observou a falta de dados estatísticos confiáveis, sub-registro e má classificação das causa de morte nos países estudados ${ }^{1}$.

De acordo com aquela publicação, o Brasil utiliza para calcular a mortalidade materna o sistema estatístico chamado Direct Sisterhood Method, que requer amostras muito grandes, e a informação gerada é considerada muito complexa para analisar. Além disso, o método não pode ser usado para monitorizar alterações de curtos prazos ou mesmo para avaliar o impacto de programas de maternidade segura ${ }^{1}$. Existem discrepâncias entre os resultados calculados pelo Ministério da Saúde no Brasil e aqueles de pesquisadores internacionais. Enquanto o Ministério da Saúde apresenta a razão de morte materna (RMM) de 64,8 por 100.000 nascidos vivos ${ }^{3}$, o Demographic and Health Surveys Analytical Report informa que para o Brasil a RMM é de $161^{4}$, mas pelos novos cálculos de estimativas da $\mathrm{OMS}^{1}$ a RMM ajustada do país é em realidade de 260 em média, com intervalo de confiança entre 160 e 370 por 100.000 nascidos vivos ${ }^{1}$. Trabalho cuidadoso realizado em Campinas, uma das melhores cidades do país, revela que o indice de subregistro de mortes maternas chegou a 37\%, com uma RMM de 45,5 por 100.000 nascidos vivos ${ }^{5}$. Na cidade de São Paulo a mortalidade materna foi de 50,3 (1995), com um índice de subnotificação de $28,2 \%{ }^{6}$. Levantamento recente (2003) no Hospital das Clínicas de Porto Alegre indica a mortalidade materna de 90 por 100 mil nascidos vivos ${ }^{7}$.

Com o propósito de contribuir para o estudo da mortalidade materna no Brasil, foi objetivo do presente trabalho relatar, no período de 75 anos de existência, o número e as causas de mortes maternas ocorridas em serviço obstétrico universitário de cidade de porte médio do sudeste brasileiro.

\section{Métodos}

\section{Informações sobre a Instituição}

A Maternidade Therezinha de Jesus é instituição filantrópica fundada em dezembro de 1926 é o serviço obstétrico mais antigo da cidade, um dos mais antigos do estado de Minas Gerais e desde 1958 mantém convênio para o ensino da Obstetrícia da UFJF. Atualmente a instituição não possui UTI neonatal, mas tem equipamento para atendimento de emergência do recém-nascido, tais como respiradores, cardioscópios, desfibriladores, oxímetros, berços de calor irradiante, incubadoras modernas e bombas de infusão; aspiração, oxigênio e ar medicinal são centralizados. O pessoal é capacitado: obstetras, residentes de obstetricia, neonatologistas e anestesiologistas estão presen- 
tes 24 horas na sala de parto. Pela freqüente falta de vagas nos hospitais terciários, a maternidade é geralmente o hospital de referência de ambulâncias que chegam de outras cidades da região, atendendo inclusive a pacientes de alto risco, embora seja classificada como de baixo risco, pelo Ministério da Saúde.

\section{Métodos de Análise}

Foram analisados todos os prontuários de 144 pacientes que foram ao óbito em um total de 164.161 internações obstétricas entre 1927 e 2001. Neste mesmo periodo ocorreram 134.111 partos e 17.755 abortos e 12.295 gestantes foram internadas para tratamento clínico. Das 144 mortes, quatro não tiveram seus prontuários disponiveis, portanto 140 foram analisadas detalhadamente. Os critérios de inclusão foram: todas as mortes que ocorreram no hospital no período estudado, consideradas apenas as mortes por causas obstétricas diretas e indiretas. Foram excluidas as pacientes que foram transferidas para UTI de outros hospitais e lá faleceram. Por informação do SUS (Comitê Municipal de Prevenção à Mortalidade Materna), nos últimos cinco anos (1997-2001), houve quatro pacientes que tiveram parto na Maternidade, foram transferidas para outro hospital e lá faleceram. Ao contrário, duas pacientes que tiveram parto em outro hospital foram transferidas para a Maternidade, resultando em dois óbitos, sendo estas incluídas neste estudo.

Calculou-se a proporção de mortes maternas por faixa etária, por paridade, por tempo de gestação e por causas obstétricas diretas e indiretas (análise estatística de três períodos de 25 anos pelo teste do $\left.\chi^{2} ; a=0,05\right)$. As causas de morte materna foram obtidas pelos diagnósticos clínicos dos prontuários e do atestado de óbito (não houve necropsias). A mortalidade materna por 100 mil nascidos vivos foi calculada por períodos de 15 anos e analisada pela técnica de amortecimento exponencial $(a=0,05)$.
Resultados

Entre 1927 e 2001 ocorreram 134.011 partos (98.646 partos vaginais e 35.365 cesarianas), que resultaram em 131.048 nascidos vivos, além de 17.755 abortos. De 1927 a 1941 a mortalidade materna foi de 1544 por cem mil nascidos vivos (Tabela 1). A partir de 1942 houve uma redução significativa para $314(\mathrm{p}<0,001)$ e no período seguinte (1957-1971) a mortalidade decresceu para $76,4$ ( $p<0,001)$. Entre 1972 e $1986(72,9$ por 100 mil nascidos vivos) e nos últimos 15 anos $(46,0$ por cem mil nascidos vivos) a mortalidade materna manteve-se estabilizada $(p>0,05)$.

Tabela 1 - Índice de mortalidade materna (IMM) de 1927 a 2001 por 100 mil nascidos vivos.

\begin{tabular}{lccc}
\hline Período & $\begin{array}{c}\text { Mortes maternas } \\
\mathbf{n}\end{array}$ & Nascidos vivos & IMM \\
\hline $1927-1941$ & 33 & 2137 & 1544,2 \\
$1942-1956$ & 34 & 10841 & $313,6^{*}$ \\
$1957-1971$ & 19 & 24861 & $76,4^{*}$ \\
$1972-1986$ & 41 & 56228 & 72,9 \\
$1987-2001$ & 17 & 36981 & 46,0 \\
\hline
\end{tabular}

${ }^{\star} p<0,001$ em relação ao período precedente.

Estudando-se a mortalidade materna em relação à idade da paciente observa-se que sua maior proporção acompanhou a época de maior atividade reprodutiva (15-39 anos) Ao longo dos três períodos de 25 anos, nota-se tendência à redução na mortalidade entre as adolescentes $(18,5 \%$ entre 1927-1951 para 12,5\% entre 1977-2001).

A influência da paridade sobre as mortes maternas é analisada na Tabela 2: considerandose o total de 75 anos de estudo, as nuliparas predominam sobre as demais, de forma significativa $(p<0,01)$. As parturientes que tiveram um ou mais partos apresentaram a mesma razão de mortalidade materna independente da paridade.

Tabela 2 - Incidência de mortes maternas por paridade entre 1927 e 2001.

\begin{tabular}{lccccccccc}
\hline \multirow{2}{*}{ Paridade } & $\mathbf{1 9 2 7}$ & -1951 & $\mathbf{1 9 5 2}$ & -1976 & $\mathbf{1 9 7 7}$ & $-\mathbf{2 0 0 1}$ & \multicolumn{2}{c}{ Total } \\
\hline 0 & $\mathbf{n}$ & $\%$ & $\mathbf{n}$ & $\mathbf{1}$ & $\mathbf{n}$ & $\%$ & $\mathbf{n}$ & $\%$ \\
1 & 17 & $44,7^{*}$ & 18 & 41,9 & 11 & 31,4 & 46 & $39,6^{*}$ \\
2 & 2 & 5,3 & 5 & 11,6 & 3 & 8,6 & 10 & 8,6 \\
3 & 9 & 23,7 & 2 & 4,6 & 9 & 25,7 & 20 & 17,2 \\
$\geq 4$ & 7 & 18,4 & 6 & 13,9 & 7 & 20,0 & 20 & 17,2 \\
Total & 3 & 7,9 & 12 & 27,9 & 5 & 4,3 & 20 & 17,2 \\
\hline
\end{tabular}

Sem informação - total: 28.

${ }^{*} p<0,01$. 
Quanto à duração da gravidez das 110 mortes maternas para as quais havia registro de tempo de gestação, 58,2\% (64 mulheres) ocorreram com gestação a termo $(p<0,001)$. Não houve diferença significativa quanto à mortalidade materna entre o grupo de abortos (13 casos ou $11,8 \%$ ) e de partos prematuros (33 casos ou 30,0\%) ( $p=0,630)$ (Tabela 3).

Considerando o momento da morte em relação ao parto, em $18 \%$ dos casos a morte ocorreu durante o parto ou cesárea e $53 \%$ no puerpério imediato. Nos demais prontuários (29\%) não há registro do momento da morte.

O estudo de 140 mortes evidencia que as causas obstétricas diretas ocorreram em 110 casos $(79,3 \%)$, enquanto que em 29 pacientes foram devidas às causas obstétricas indiretas $(20,7 \%)$. No primeiro quarto de século as causas obstétricas diretas mais freqüentes (Tabela 4) foram infecção puerperal $(34,9 \%)$ e eclampsia $(34,9 \%)$, seguidas da ruptura uterina intraparto $(14,0 \%)$ e aborto $(7,0 \%)$. No segundo período foram mais freqüentes a hemorragia pré-parto $(41,7 \%)$, a eclampsia
$(36,1 \%)$ e as rupturas uterinas $(11,1 \%)$. No terceiro quarto de século as causas mais freqüentes foram: 11 hemorragias $(35,5 \%)$, sendo oito no pré-parto e três após o parto, seguidas dos abortos infectados $(25,8 \%)$ e da pré-eclampsia/eclampsia $(25,8 \%)$.

Quanto às causas obstétricas indiretas entre 1927 e 1951, a cardiopatia com quatro casos foi responsável por quase metade das mortes, seguida de outras patologias pré-existentes, como três casos de insuficiência renal aguda, um caso de câncer de vagina e um de tuberculose pulmonar. No período seguinte as mais freqüentes foram cardiopatia e acidente vascular cerebral (AVC), com cinco casos cada ou $35,7 \%$, dois casos de embolia pulmonar e três por outras patologias (leucemia, pneumonia aguda na admissão e íleo paralítico pós-cesárea). No terceiro quarto de século a maior incidência foi de AVC (três casos ou 42,8\%), seguida de cardiopatia (dois casos), um caso de embolia pulmonar e um caso de morte por acidente em anestesia geral em curetagem por mola hidatiforme. Durante os 75 anos de estudo as principais causas de mortes

Tabela 3 - Incidência de mortes maternas por tempo de gestação (1927-2001).

\begin{tabular}{lccccccc}
\hline \multirow{2}{*}{ Semanas } & \multicolumn{2}{c}{$1927-1951$} & \multicolumn{2}{c}{$1952-1976$} & \multicolumn{2}{c}{$1977-2001$} & \multicolumn{2}{c}{ Total } \\
& $\mathbf{n}$ & $\%$ & $\mathbf{n}$ & $\%$ & $\mathbf{n}$ & $\%$ & $\mathbf{n} \%$ \\
\hline 20 & 3 & 38,3 & 3 & 7,3 & 7 & 21,2 & 131,8 \\
$21-36$ & 11 & 30,5 & 12 & 29,3 & 10 & 30,3 & 3330,0 \\
$37-41$ & 22 & $61,1^{*}$ & 26 & $63,4^{*}$ & 16 & $48,5^{*}$ & $6458,2^{*}$ \\
Total & 36 & 100,0 & 41 & 100,0 & 33 & 100,0 & 110100,0 \\
\hline
\end{tabular}

Sem informação - total: 34

*37-41 semanas vs demais grupos $(p<0,01)$

Tabela 4 - Incidência das causas obstétricas diretas de mortes maternas entre 1927 e 2001.

\begin{tabular}{|c|c|c|c|c|c|c|c|c|}
\hline \multirow{2}{*}{ Causas } & \multicolumn{2}{|c|}{$1927-1951$} & \multicolumn{2}{|c|}{$1952-1976$} & \multicolumn{2}{|c|}{$1977-2001$} & \multicolumn{2}{|c|}{ Total } \\
\hline & $\mathrm{n}$ & $\%$ & $\mathrm{n}$ & $\%$ & $\mathrm{n}$ & $\%$ & $\mathrm{n}$ & $\%$ \\
\hline $\begin{array}{l}\text { Pré-eclampsia/ } \\
\text { eclampsia }\end{array}$ & 15 & 34,9 & 13 & 36,1 & 8 & 25,8 & 36 & $32,7^{*}$ \\
\hline Infecção puerperal & 15 & 34,9 & 0 & 0,0 & 2 & 6,4 & 17 & $15,4^{*}$ \\
\hline Hemorragia pré-parto & 2 & 4,6 & 15 & 41,7 & 8 & 25,8 & 25 & $22,7^{*}$ \\
\hline $\begin{array}{l}\text { Hemorragia } \\
\text { pós-parto }\end{array}$ & 1 & 2,3 & 3 & 8,3 & 3 & 9,7 & 7 & 6,4 \\
\hline Aborto & 3 & 7,0 & 1 & 2,8 & 8 & 25,8 & 12 & 10,9 \\
\hline Ruptura de útero & 6 & 14,0 & 4 & 11,1 & 1 & 3,2 & 11 & 10,0 \\
\hline Outras & 1 & 2,3 & 0 & 0,0 & 1 & 3,2 & 2 & 1,8 \\
\hline Total & 43 & 100,0 & 36 & 100,0 & 31 & 100,0 & 110 & 100,0 \\
\hline
\end{tabular}


obstétricas indiretas foram cardiopatia, seguida de AVC e embolia pulmonar.

Analisando mais detalhadamente as principais causas obstétricas diretas verifica-se que a infecção puerperal no primeiro período (1927-51) contribuiu com 15 mortes maternas, sendo 14 puerperais e uma iniciada no pré-parto. No segundo período não houve morte materna por processo infeccioso. No terceiro período houve duas mortes, sendo uma por amniotite pré-parto e uma internação pós-parto com infecção puerperal. A mortalidade materna por infecção teve forte redução nos últimos 50 anos. Em relação à eclampsia, no primeiro período (1927-51) ocorreram 15 mortes maternas, no segundo período (1952-76) houve 12 mortes por eclampsia e uma por pré-eclampsia grave. Nos últimos 25 anos, ocorreram três mortes por eclampsia e cinco por pré-eclampsia grave, sendo que destas, duas foram associadas a descolamento prematuro de placenta (DPP) e uma com coagulação intravascular disseminada (CIVD).

Não houve morte materna por pré-eclampsia /eclampsia nos últimos 15 anos. Em relação às hemorragias, no primeiro período (1927-51) ocorreram seis casos de ruptura uterina intraparto com hemorragia, dois casos de placenta prévia (PP) e um caso de hemorragia pós-parto por atonia uterina. No segundo período foram nove casos de $\mathrm{PP}$, seis de DPP, quatro rupturas uterinas e três de hemorragia pós-parto. Houve coincidência de CIVD em três pacientes associadas com DPP e PP. Nos últimos 25 anos, houve três pacientes com PP, seis com DPP, uma com ruptura uterina e três com hemorragia pós-parto, com associação de CIVD em quatro e de placenta acreta em quatro. Análise mais profunda dos casos de aborto, considerando a incidência sobre o total das internações obstétricas, mostra que no primeiro período ocorreu incidência de $3,7 \%$ de abortos, com três mortes. No segundo período houve incidência de $7,2 \%$ de aborto infectado, com apenas uma morte materna, e no terceiro período, oito mortes, em $13,5 \%$ de abortos, sendo cinco por aborto infectado, uma mola hidatiforme e duas mortes com perfurações uterinas durante curetagem por aborto incompleto, com histerectomia subseqüente e CIVD.

Após publicação anterior com estudo de 60 anos de mortes maternas no mesmo hospital ${ }^{8}$, a análise dos dados dos últimos 15 anos de assistência obstétrica mostrou que não houve morte materna por pré-eclampsia/eclampsia nem por infecção, e as principais causas de mortes maternas continuam a ser hemorragia periparto e aborto, e manutenção das causas obstétricas indiretas.

A avaliação das mortes maternas associadas ao tipo de parto mostrou que as principais causas de indicação de cesárea que resultaram em morte materna foram as hemorragias (DPP, PP e atonia uterina), com 17 casos, seguidos de eclampsia (10 casos), porém enquanto a pré-eclampsia/eclampsia vem diminuindo proporcionalmente, as hemorragias periparto vêm se mantendo como a principal causa. Como os números dos primeiros 25 anos são pequenos, fez-se a comparação de risco relativo (RR) de morte materna por cesárea ou parto vaginal dos últimos 50 anos (Tabela 5). O risco de morte materna, relacionado exclusivamente à cesárea, foi 2,4 vezes maior que por parto via vaginal. No entanto, o exame mais cuidadoso dos 35 casos de morte materna relacionados à cesárea mostra que em 25 a indicação de cirurgia era inevitável e apenas 10 mortes maternas poderiam ser atribuídas exclusivamente à operação cesariana, com uma redução do RR cesárea/parto vaginal para 0,6. Estas dez mortes tiveram como causa a hemorragia pós-cesárea sem sangramento prévio (dois casos), PP marginal sem sangramento (dois casos), apresentação pélvica em nulipara com AVC (dois casos), embolia pulmonar pós-operatória, CIVD com hemorragia pós-operatória, feto morto em paciente com AVC e íleo paralítico pós-operatório.

Tabela 5 - Índice de morte materna (IMM) e risco relativo (RR) com cesárea ou parto vaginal nos últimos 50 anos (1952-2001) com e sem expurgo estatístico.

\begin{tabular}{lccc}
\hline Parâmetros & Partos vaginais & Cesáreas & RR \\
\hline Mortes maternas & 41 & 35 & \\
Nascidos vivos & 88.397 & 34.732 & \\
IMM & 46,4 & 100,8 & 2,4 \\
IMM com expurgo & 46,4 & 28,8 & $0,6^{*}$ \\
\hline${ }^{*}$ Consideradas somente 10 mortes maternas relacionadas ao ato cirúrgico
\end{tabular}

\section{Discussão}

A estimativa da Organização Mundial da Saúde para a mortalidade materna em todo o mundo foi de 397 por cem mil nascidos vivos, observando enormes diferenças entre regiões ${ }^{9}$. Nos Estados Unidos da América, por exemplo, os Centers for Disease Control and Prevention (CDC) de Atlanta informam que a mortalidade materna aumentou de 10,3 em 1991 para 12,9 por cem mil nascidos vivos, em $1997^{10}$. A diferença abissal entre a mortalidade materna em países de terceiro mundo e dos desenvolvidos pode ser notada num estudo comparativo entre hospitais americanos e da Zâmbia. A mortalidade materna foi de 20,4 para os primeiros e de 1540 por cem mil nascidos vivos no segundo ${ }^{11}$. Na Índia (hospital de Nova Delhi) o índice era de $468 \mathrm{em}$ 1989 e de 190 por mil nascidos vivos em $1998^{12}$.

No Brasil, a RMM estimada para o ano 2000 foi de 64,3 . Porém a subenumeração de mortes maternas exige grandes correções, especialmente nas 
regiões do Norte e Nordeste do país ${ }^{13}$. No Hospital de Clínicas de Porto Alegre, analisando-se mortes relacionadas à gestação e puerpério até um ano após o parto, a mortalidade materna dos casos nascidos no hospital foi de 90, ao passo que, quando o parto foi em outro hospital e trazido para o HC, este índice foi de 109 por 100 mil nascidos vivos ${ }^{7}$. No Recife, a RMM foi de 75,5 e o percentual de sub-registro encontrado foi de $27,8 \%{ }^{14}$. No Hospital Miguel Couto do Rio de Janeiro, em estudo de 10 anos, a mortalidade materna foi de 177 por cem mil nascidos vivos ${ }^{15}$. Verifica-se, portanto, que há disparidade entre hospitais de diferentes estados no mesmo país. No presente estudo o índice de mortalidade materna hospitalar calculado por 100.000 nascidos vivos foi reduzido de 1544 no período entre 1927 e 1941 para 76,4 no período de 1957-71, no entanto desde 1972 não se tem conseguido reduzir a mortalidade de maneira significativa, sendo ainda muito elevada. Em Juiz de Fora, entre 1996 e 2003, o indice de mortalidade materna dos hospitais foi de 125,1 , sendo que na Maternidade Therezinha de Jesus foi de 55,9 e nos demais hospitais foi de 147,4 por cem mil nascidos vivos $^{16}$. Podemos concluir, portanto, que o índice de mortalidade materna da Maternidade Therezinha de Jesus, embora elevado, está bem posicionado entre outros hospitais da cidade e do país.

Considerando a mortalidade materna por faixas etárias, observou-se nos Estados Unidos da América um risco aumentado para mulheres negras, mais idosas e sem cuidados prenatais ${ }^{10}$, e que a RMM aumenta com a idade, indo de 2,7 na faixa de 35 anos para 7,9 quando acima de 40 anos, indicando necessidade de cuidados especiais nesta faixa etária ${ }^{17}$.

Estudo de mais de 850 mil mulheres do Centro Latino-Americano para Perinatologia de Montevidéu indica que adolescentes com 15 anos de idade ou menos têm maior risco de morte materna ${ }^{18}$. No Brasil, observou-se que o grupo etário de 20-29 anos tem risco mais elevado, ocorrendo nesta faixa $48 \%$ dos óbitos ${ }^{19}$. No presente trabalho observou-se grande incidência de morte materna na época de maior atividade reprodutiva, isto é, entre 20 e 39 anos. A incidência de morte materna em jovens adolescentes entre 15-19 anos foi também elevada durante todo o estudo, confirmando os trabalhos citados ${ }^{18,19}$.

Em relação ao número de partos anteriores, julgando a importância da paridade com morbidade da eclampsia com sindrome de HELLP, observou-se que na multípara com eclampsia o quadro é mais grave que nas nuliparas ${ }^{20}$.

Quanto ao tempo de gestação, a maior incidência de mortes maternas foi no grupo de gestação a termo, não havendo diferença significativa entre o grupo de abortos e de partos prematuros. Em relação ao momento da morte materna, do mesmo modo que foi observado em Niterói ${ }^{19}$, as mortes ocorreram em sua maioria no puerpério imediato.

Quanto às causas de mortalidade materna, em estudo de autopsias efetuadas em 45\% dos casos de morte materna na Índia, encontraram hipertensão com complicações de CIVD, hemorragias de diferentes órgãos, tromboembolismo e anemia como as principais causas obstétricas diretas ${ }^{21}$. Na França e no Reino Unido, o embolismo de líquido amniótico é considerado como a terceira e a quinta causa mais freqüente, respectivamente, de morte materna ${ }^{22}$, ao passo que nos Estados Unidos as principais causas de morte materna foram o embolismo, hemorragia e outras condições médicas ${ }^{10}$.

No Brasil o embolismo é pouco diagnosticado, provavelmente pelo baixo índice de necropsias no país. Em Campinas as causas obstétricas diretas foram da ordem de $75 \%$ e muitas poderiam ser evitadas ${ }^{5}$. No Recife, as causas obstétricas diretas mais freqüentes foram hipertensão (19\%), hemorragia $(16 \%)$ e infecção $(11 \%)$, sendo consideradas evitáveis em $82 \%$ dos $\operatorname{casos}^{14}$. No presente estudo as causas obstétricas diretas mais freqüentes nos 75 anos analisados foram pré-eclampsia/eclampsia, seguida de hemorragia pré-parto, infecção puerperal, aborto infectado, ruptura uterina intraparto e hemorragia pós-parto. No entanto, considerando o periodo de 1977 a 2001, as causas mais freqüentes foram hemorragia pré-parto e pós-parto, seguidas pelo aborto e pré-eclampsia. A hemorragia é atualmente a maior causa de morte materna, com associação muito freqüente de DPP, pré-eclampsia/ eclampsia, coagulação intravascular disseminada e PP acreta. Diversos casos foram de pacientes recebidas de centros menores já com hemorragia de vários dias, e a demora entre a solicitação de uma transfusão de sangue e a sua realização foi problema de dificil solução.

A análise detalhada das principais causas obstétricas diretas observadas na Maternidade Therezinha de Jesus mostra que a infecção como causa de morte materna foi progressivamente reduzida nos últimos 50 anos. A alta mortalidade por eclampsia, nos primórdios das observações teve, também, nos últimos 25 anos, significativa redução com o uso de esquemas mais rígidos, não só do emprego judicioso e amplo do magnésio, mas também de atitudes mais agressivas dos obstetras, buscando interromper a gestação sempre que possivel, uma vez que este é o único tratamento definitivo atual desta doença. Eliminando as mortes maternas por eclampsia, logicamente apareceu como importante a pré-eclampsia grave, associada não raras vezes ao DPP e CIVD. 
Preocupação atual é o aumento de mortalidade por aborto, especialmente os abortos clandestinos em adolescentes. No Hospital Miguel Couto do Rio de Janeiro a mortalidade materna foi de 177 por cem mil nativivos, mas a incidência de mortes por aborto foi de $47 \%$ do total, tendo aumentado $172 \%$ no período de 10 anos, o que foi atribuído à falta de planejamento familiar e a condições socioeconômicas precárias ${ }^{15}$. A Alta mortalidade materna por aborto é também relatada na Finlândia: a mortalidade materna foi de 51,9 para aborto espontâneo e de 83,1 (por 100 mil nascidos vivos) para aborto induzido, chamando a atenção que muitas mortes ocorrem neste período de observação sem o devido registro hospitalar ${ }^{23}$.

Entre as causas obstétricas indiretas, Ávila et al. ${ }^{24}$ (INCOR - São Paulo), estudando cardiopatias em gestantes, verificaram que $76 \%$ delas não tiveram eventos cardiovasculares e que a insuficiência cardiaca congestiva apareceu em $12,3 \%$, as arritmias cardiacas em $6 \%$, tromboembolismo em 1,9\%, angina em 1,4 e outras, em menor número, chegando à conclusão que gravidez em cardiopata se correlaciona fortemente com a doença subjacente, com consideráveis indices de morbidade e de mortalidade, e que cuidados prenatais e estratificação precoce dos riscos na gestação são fundamentais para a gestante cardiopata melhorar seu prognóstico. No presente trabalho, no primeiro quarto de século a causa mais freqüente de morte materna indireta foi a cardiopatia, seguida de insuficiência renal aguda. No segundo período as causas foram cardiopatia, AVC e tromboembolia. Nos últimos 25 anos a maior incidência foi de AVC, seguida de cardiopatia. Ao longo dos 75 anos as principais causas indiretas de morte foram por cardiopatia, seguida de AVC e tromboembolia.

Correlacionando-se os dados atuais com publicação de estudo de 60 anos anteriores ${ }^{8}$, verifica-se que nos últimos 15 anos não houve morte por préeclampsia/eclampsia nem por infecção puerperal e as principais causas continuam a ser hemorragia periparto, aborto e manutenção das causas obstétricas indiretas. Em relação ao tipo de parto, ao longo dos anos a mortalidade materna teve redução significativa tanto para partos vaginais como abdominais, mas ainda é muito elevada ${ }^{5}$. No Reino Unido o RR de morte materna associada ao parto vaginal foi 1,5 vezes maior do que com a cesárea eletiva ${ }^{25}$. Analisando-se dados do estado de Massachusetts para associação cesárea/morte materna entre mais de 600 mil partos, a RMM da cesárea foi de 5,8, ao passo que no parto vaginal foi de 10,8 por $100 \mathrm{mil}$ nascidos vivos, concluindo-se que o RMM por cesárea é pequeno ${ }^{26}$. Na presente análise da associação de mortes maternas e o tipo de parto observa-se que as principais indicações de cesarianas foram hemorragias e a pré-eclampsia/eclampsia. Enquanto esta vem diminuindo progressivamente, a hemorragia periparto vem se mantendo como forte indicação da cesárea, freqüentemente inevitável. Verifica-se que a relação de mortes maternas em cesárea é 2,4 vezes mais freqüente do que em parto vaginal. Porém, com análise cuidadosa de 10 mortes atribuíveis exclusivamente ao ato cirúrgico, quando a indicação da cesárea é absoluta e sem a menor possibilidade de um parto por via vaginal, o RR de morte materna por cesárea é menor do que a mortalidade associada ao parto transpélvico $(R R=0,6)$, confirmando dados do estado de Massachusetts ${ }^{26}$.

Em conclusão, apesar de sua redução progressiva, a mortalidade materna ainda é muito elevada e muitas mortes são evitáveis. Importante é ter maior atenção para os partos de pacientes de faixa etária abaixo de 19 anos e nuliparas. A mortalidade por infecção puerperal e pré-eclampsia/ eclampsia podem ser minimizadas com assistência atualizada. As hemorragias do pré- e do pós-parto, causas mais freqüentes de morte materna, devem ser cuidadosamente monitorizadas e não pode haver demora no atendimento nem postergar decisão importante de intervir e realizar cesárea mais prontamente, especialmente nas indicações inevitáveis. É obrigatório rever o problema dos abortos clandestinos, da legislação concernente e orientar e fornecer métodos contraceptivos a toda a população para evitar a gravidez indesejada.

\section{Agradecimentos}

Professora Jane Azevedo Silva, Departamento de Estatística - Instituto de Ciências Exatas - UFJF, pela análise estatística.

\section{Referências}

1. WHO. UNICEF. UNFPA. Maternal mortality in 2000: estimates developed by WHO, UNICEF and UNFPA. Geneva: World Health Organization; 2004.

2. Tsu VD, Shane B. New and underutilized technologies to reduce maternal mortality: call to action from a Bellagio workshop. Int J Gynecol Obstet. 2004;85 Suppl 1:S83-93.

3. Ministério da Saúde. Secretaria de Políticas de Saúde. Área Técnica de Saúde da Mulher. Manual dos comitês de mortalidade materna. $2^{\text {a }}$ ed. Brasília: Ministério da Saúde; 2002.

4. Stanton C, Abderrahim N, Hill K. DHS maternity mortality indicators: an assessment of data quality and implications for data use. Calverton: Macro 
International Inc.; 1997. (Demographic and Health Surveys Analytical Report, 4).

5. Cecatti JG, Faundes A, Surita FG. Maternal mortality in Campinas: evolution, under-registration and avoidance. Rev Paul Med. 1999;117(1):5-12.

6. Boyaciyan K, Marcus PAF, Vega CEP, Barbosa SA, Pazero LC. Mortalidade materna na cidade de São Paulo de 1993 a 1995. Rev Bras Ginecol Obstet. 1998;20(1):13-8.

7. Ramos JGL, Martins-Costa S, Vettorazzi-Stuczynski J, Brietzke E. Morte materna em hospital terciário do Rio Grande do Sul - Brasil: um estudo de 20 anos. Rev Bras Ginecol Obstet. 2003;25(6):431-6.

8. Andrade ATL, Andrade DN, Faza MC, Cotta MCMB, Clark LH, Araújo DAC. Mortalidade materna: estudo de 60 anos. Rev Bras Ginecol Obstet. 1988;10(4):205-10.

9. Hill K, AbouZhar C, Wardlaw T. Estimates of maternal mortality for 1995. Bull World Health Organ. 2001;79(3):182-93.

10.Berg CJ, Chang J, Callaghan WM, Whitehead SJ. Pregnancy-related mortality in the United States, 1991-1997. Obstet Gynecol. 2003;101(2):289-96.

11.Kilpatrick SJ, Crabtree KE, Kemp A, Geller S. Preventability of maternal deaths: comparison between Zambian and American referral hospitals. Obstet Gynecol. 2002;100(2):321-6.

12.Baul MK, Manjusha. Maternal mortality - a ten-year study. J Indian Med Assoc. 2004;102(1):18-9, 25.

13. Formiga Filho JFN, Parras AA, Bueno H, Reis PAK, Chaves SVR. Mortalidade materna. In: Corrêa MD, Melo VH, Aguiar RALP, Corrêa Júnior MD, editores. Noções práticas de obstetrícia. $13^{\mathrm{a}}$ ed. Belo Horizonte: Editora Coopmed; 2004. p. 427-35.

14. Costa AAR, Ribas MSSS, Amorim MMRA, Santos LC. Mortalidade materna na cidade do Recife. Rev Bras Ginecol Obstet. 2002;24(7):455-62.

15.Laguardia KD, Rotholz MV, Belfort P. A 10-year review of maternal mortality in a municipal hospital in Rio de Janeiro: a cause for concern. Obstet Gynecol. 1990;75(1):27-32.

16.Comitê Municipal de Prevenção a Mortalidade Materna em Juiz de Fora. Relação anual das mortes maternas com passagem pela Maternidade There-zinha de Jesus, jan 1996-dez 2003. Juiz de Fora: SINASC/DEPI/DSSDA/JF; 2003.

17.Callaghan WM, Berg CJ. Pregnancy-related mortality among women aged 35 years and older, United States, 1991-1997. Obstet Gynecol. 2003;102(5 Pt 1): 1015-21.

18.Conde-Agudelo A, Belizan JM, Lammers, C. Maternal-perinatal morbidity and mortality associated with adolescent pregnancy in Latin America: cross-sectional study. Am J Obstet Gynecol. 2005; 192(2):342-9.

19.Arkader J. Mortalidade materna [tese]. Niterói: Universidade Federal Fluminense; 1993.

20.Isler CM, Rinehart BK, Terrone DA, May WL, Magann EF, Martin JN Jr. The importance of parity to major maternal morbidity in the eclamptic mother with HELLP syndrome. Hypertens Pregnancy. 2003;22(3):287-94.

21.Kavatkar AN, Sahasrabudhe NS, Jadhav MV, Deshmukh SD. Autopsy study of maternal deaths. Int J Gynaecol Obstet. 2003;81(1):1-8.

22.Tuffnell DJ. Amniotic fluid embolism. Curr Opin Obstet Gynecol. 2003;15(2):119-22.

23. Gissler M, Berg C, Bouvier-Colle MH, Buekens P. Pregnancy-associated mortality after birth, spontaneous abortion, or induced abortion in Finland, 1987-2000. Am J Obstet Gynecol. 2004;190(2):422-7.

24.Avila WS, Rossi EG, Ramires JA, Grinberg M, Bortolotto MR, Zugaib M, et al. Pregnancy in patients with heart disease: experience with 1,000 cases. Clin Cardiol. 2003;26(3):135-42.

25. Lilford RJ, van Coeverden de Groot HA, Moore PJ, Bingham P. The relative risks of caesarean section (intrapartum and elective) and vaginal delivery: a detailed analysis to exclude the effects of medical disorders and other acute pre-existing physiological disturbances. Br J Obstet Gynaecol. 1990;97(10):883-92.

26.Sachs BP, Yeh J, Acker D, Driscoll S, Brown DA, Jewett JF. Cesarean section-related maternal mortality in Massachusetts, 1954-1985. Obstet Gynecol. 1988;71(3 Pt 1):385-8. 